\title{
Severe tricuspid regurgitation 14 years after diagnosis of "transient neonatal tricuspid regurgitation"
}

\author{
D Boshoff, L Mertens, M Gewillig
}

\begin{abstract}
A 14 year old girl presented with severe tricuspid regurgitation after she was diagnosed with "transient tricuspid regurgitation of the newborn". In the neonatal period she had presented with severe tricuspid regurgitation without an obvious underlying anatomical cause. This spontaneously regressed during the first months of life. She was dismissed from follow up at the age of 5 years after complete normalisation of the clinical and echocardiographic examination. The subsequent evolution and management of the patient, as well as the possible pathogenesis responsible for the unusual clinical course, is discussed. This case stresses the importance of long term follow up of patients with transient tricuspid regurgitation.

(Heart 2001;86:88-90)
\end{abstract}

Keywords: neonatal cardiomyopathy; regurgitation; tricuspid valve

A 14 year old female patient presented in our outpatient clinic with a history of progressive exercise intolerance during the preceding six months. In the neonatal period she was diagnosed with transient tricuspid regurgitation. At the time she presented with severe cyanosis and cardiomegaly. She was included in the report of our group published on this condition. ${ }^{1}$ The degree of neonatal tricuspid regurgitation resulted in functional pulmonary atresia. $^{2-5}$ Echocardiography in the neonatal period showed a structurally normal heart and no obvious morphological anomaly of the tricuspid valve. Within three months the tricuspid regurgitation and cyanosis regressed progressively, as illustrated by the regression of the massive cardiomegaly on chest $x$ ray (fig 1 ). When she was six months of age the clinical and Doppler echocardiographic findings were within normal limits. Routine yearly clinical and echocardiographic findings remained normal for five years and therefore the patient was discharged from further cardiac follow up.

On clinical examination at 14 years the patient looked pale with a distinct flushed face without cyanosis. A prominent right ventricular heave was palpable at the lower left sternal border. A grade 3/6 holosystolic murmur and low pitched diastolic rumble were heard along the lower left sternal border. The ECG showed right atrial enlargement, right axis deviation, and right bundle branch block. The chest $x$ ray showed massive cardiomegaly (cardiothoracic ratio of 0.85) (fig 2A). Echocardiography showed giant dilatation of the right atrium and right ventricle and gross tricuspid regurgitation. The tricuspid annulus was severely enlarged $(53 \mathrm{~mm})$. The valve leaflets were normally implanted without significant apical displacement of the septal and posterior leaflets. The leaflets seemed slightly echodense with somewhat short chordae tendineae. The pulmonary valve opened normally with normal blood flow velocities in the pulmonary trunk. The atrial septum was intact. Right ventricular function was moderately depressed as assessed visually.
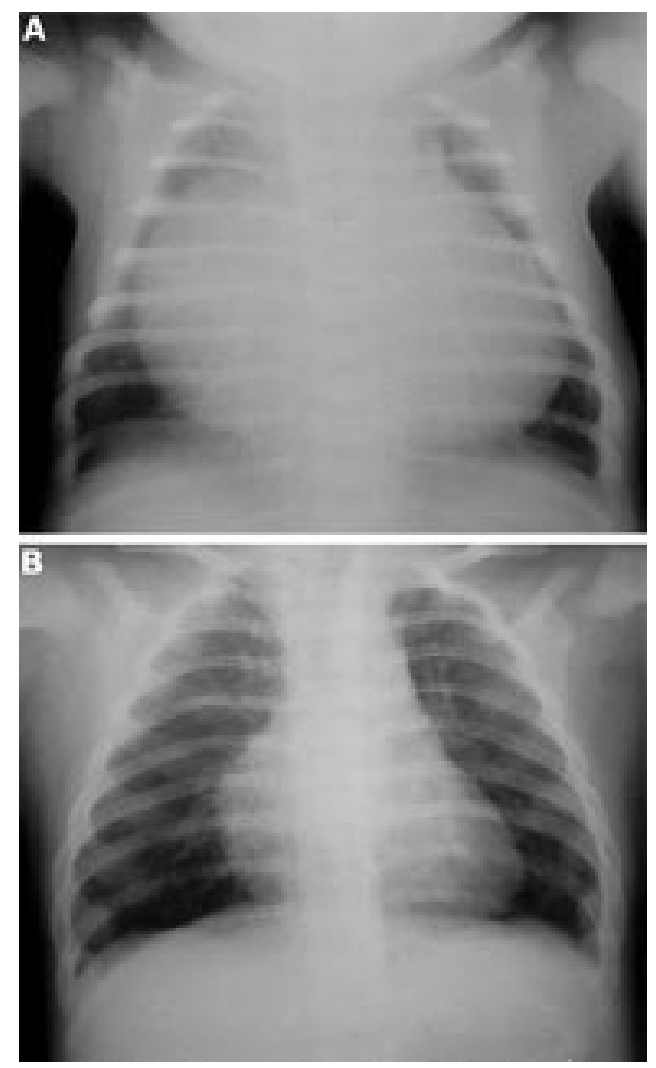

Figure 1 (A) Chest $x$ ray when the patient was 1 day old showing massive cardiomegaly caused by dilatation of the right atrium and ventricle. (B) At age 3 months only slight right atrial dilatation persisted. Reprinted from Gewillig et al with permission of the publisher. 

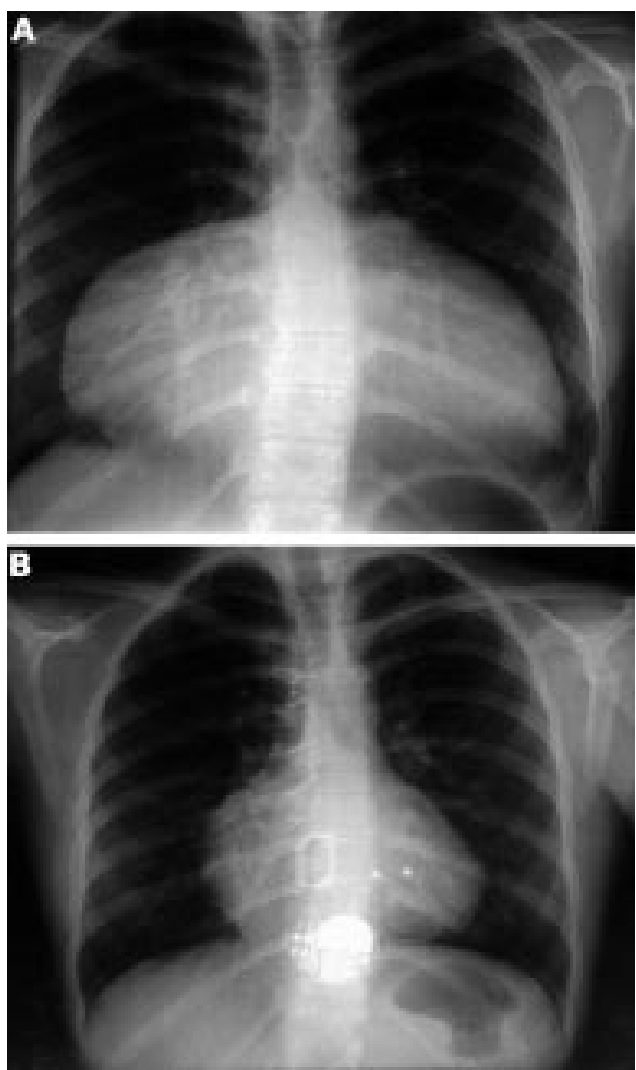

Figure 2 (A) Chest $x$ ray on re-presentation at the age of 14 years, showing massive cardiomegaly (cardiothoracic ratio 0.85 ) with predominant right atrial enlargement. (B) One month after surgery the size of the heart has decreased dramatically (cardiothoracic ratio 0.5).

Cardiac catheterisation confirmed severe tricuspid regurgitation; right atrial pressures were increased (mean $13 \mathrm{~mm} \mathrm{Hg}$ ) with "ventricularisation" of the pressure curve (prominent V wave). The mixed venous saturation was low $(52 \%)$, indicating a low cardiac output. Normal pulmonary artery pressures were confirmed. Angiography showed normal anatomy of the pulmonary arteries.

Surgery was performed in an attempt to repair the tricuspid valve and to reduce the right atrial volume. During surgery the tricuspid annulus appeared to be severely dilated. The septal leaflet had a short chordal attachment to the septum with the anterior leaflet appearing small, thin, and with multiple fenestrations. The valve was found to be unsuitable for repair and was replaced with a $33 \mathrm{~mm}$ bioprosthesis, and a reduction right atrioplasty was performed. Third degree atrioventricular block persisted for more than 10 days after cardiac surgery necessitating implantation of a permanent pacemaker.

At follow up one month after operation, there was a dramatic decrease in the size of the heart on $x$ ray (fig $2 \mathrm{~B}$ ). The bioprosthetic valve function was normal, but echocardiographically moderate right atrial and right ventricular enlargement persisted, with distinct right ventricular dysfunction. The patient's exercise capacity notably improved.

\section{Discussion}

Severe tricuspid regurgitation in the neonatal period can be caused by various anatomical anomalies of the tricuspid valve, ${ }^{16}$ but it can also be secondary to severe pulmonary hypertension or obstruction of the right ventricular outflow tract. ${ }^{7}$ Isolated transient tricuspid regurgitation without an obvious anatomical and haemodynamic basis has also been described. ${ }^{8}$ Antenatal events that cause increased pressure in the right ventricle may explain this clinical entity. It has been shown that fetal closure of the ductus arteriosus causes increased pulmonary arterial pressure, right ventricular enlargement, and tricuspid valve regurgitation. This mostly improves and even resolves during the first weeks of life. ${ }^{45}$

Our patient presented during the neonatal period with the clinical picture of functional pulmonary atresia caused by massive tricuspid regurgitation and "normal" high perinatal pulmonary vascular resistance. We previously reported her case as an example of "transient tricuspid regurgitation". ${ }^{1}$ Other than a slightly thickened tricuspid valve, neonatal Doppler echocardiographies excluded gross anomalies of the tricuspid valve such as Ebstein's anomaly or severe dysplasia of the tricuspid valve. Over a period of several weeks, as the pulmonary vascular resistance decreased and the right ventricle recuperated, her haemodynamic condition improved progressively, and at the ages of 3 and 6 months she had normal clinical and Doppler echocardiographic examinations. At the age of 14 years our patient once more presented with severe tricuspid regurgitation and massive dilatation of the right heart.

Echocardiographically the tricuspid valve leaflets appeared slightly thickened and the chordae tendineae seemed remarkably shortened. This is an unusual evolution after transient tricuspid regurgitation. Various hypotheses can be put forward to explain this observation. It is possible that with time the tricuspid valve started to leak again because of progressive right ventricular dysfunction long after an initial perinatal insult. Sustained perinatal right ventricular overload could have caused subendocardial myocardial damage involving the papillary muscles. ${ }^{9}$ When right ventricular afterload decreased postnatally, ventricular function initially improved resulting in decreased tricuspid regurgitation. The "scarred" ventricle continued to function relatively normally but progressively became dysfunctional after many years. This resulted in recurrence of tricuspid regurgitation, which further caused ventricular dysfunction. A second possibility is that the growth of the tricuspid valve was disturbed. A striking finding on echocardiography was the short chordae tendineae, which seemed to have grown inadequately over the preceding years. It is, however, difficult to assess the adequacy of the length of the chordae in the context of a severely dilated right ventricle. Another, less probable, explanation is that our patient did have mild Ebstein's disease. Although the single most diagnostic feature - namely, downward displacement of the septal leaflet-was absent, the surgeon reported some features suggestive of Ebstein's anomaly: a severely dilated annulus, "Ebstein-like" adhesion of the 
septal leaflet, and a thin anterior leaflet with multiple fenestrations. ${ }^{10}$ Anatomical and functional severity in Ebstein's anomaly are not necessarily related and newborns with isolated Ebstein's anomaly often show spontaneous improvement as the pulmonary vascular resistance decreases. ${ }^{10}$

From this case we conclude that patients with transient neonatal tricuspid regurgitation, without an obvious anatomical and haemodynamic basis, should be followed up during growth for the possible development of late tricuspid regurgitation.

Sponsored in part by The Belgian Foundation for Research in Paediatric Cardiology

1 Gewillig M, Dumoulin M, Van der Hauwaert LG. Transient neonatal tricuspid regurgitation: a Doppler echocardiographic study of three cases. Br Heart f 1988;60:446-51.

2 Smallhorn JF, Izukawa T, Benson L, et al. Noninvasive recognition of functional pulmonary atresia by echocardiography. Am f Cardiol 1984;54:925-6.
3 Lee CL, Hsieh KS, Huang TC, et al. Recognition of functional pulmonary atresia by color Doppler echocardiography. Am f Cardiol 1999;83:987-8.

4 Berry TE, Muster AJ, Paul MH. Transient neonatal tricuspid regurgitation: possible relation with premature closure of the ductus arteriosus. 7 Am Coll Cardiol 1983;2:117882.

5 Leal SD, Cavalle-Garrido T, Ryan G, et al. Isolated ductal closure in utero diagnosed by fetal echocardiography. $A m \mathcal{F}$ Perinatol 1997;14:205-10.

6 Barr PA, Celermajer JM, Bowdler JD, et al. Severe congenital tricuspid incompetence in the neonate. Circulation 1974;49:962-7.

7 Davignon AL, Greenwold WE, Dushane JW, et al. Congenital pulmonary atresia with intact ventrical septum: clinopathologic correlation of two anatomic types. $\mathrm{Am}$ Heart f 1961;62:591-602.

8 Boucek RJ, Graham TJ, Morgan JP, et al. Spontaneous resolution of massive congenital tricuspid insufficiency. Circulation 1976;54:795-800

9 Zenker M, Klinge J, Krueger C, et al. Severe pulmonary hypertension in a neonate caused by premature closure of the ductus arteriosus following maternal treatment with diclofenac: a case report. F Perinat Med 1998;26:231-34.

10 MacLellan-Tobert SG, Porter CJ. Ebstein's anomaly of the tricuspid valve. In: Garson A, ed. The science and practice of pediatric cardiology, 2nd edn. Baltimore: Williams \& Wilkens, 1998:1303-15.

\section{"Kissing abscess" of the anterior mitral valve leaflet from a vegetation on the non-coronary aortic cusp}

A 56 year old obese woman with no significant past medical illness was referred to the cardiac centre with a one week history of worsening shortness of breath on exertion and a one year history of fatigue. There was no history of fever, night sweats or loss of weight. On examination, she was apyrexial and mildly clubbed; there was a splinter haemorrhage on the right middle finger and she was pale. Cardiac auscultation revealed an ejection systolic murmur and an early diastolic murmur in the aortic area. Urine analysis showed microscopic haematuria. The erythrocyte sedimentation rate was $119 \mathrm{~mm} /$ hour, the C-reactive protein was $62 \mathrm{mg} / \mathrm{l}$, and the plasma creatinine was $137 \mu \mathrm{mol} / 1$. The patient's ECG and chest $x$ ray were normal. A transthoracic echocardiogram showed a $1.5 \mathrm{~cm}$ mobile vegetation attached to the non-coronary cusp of the

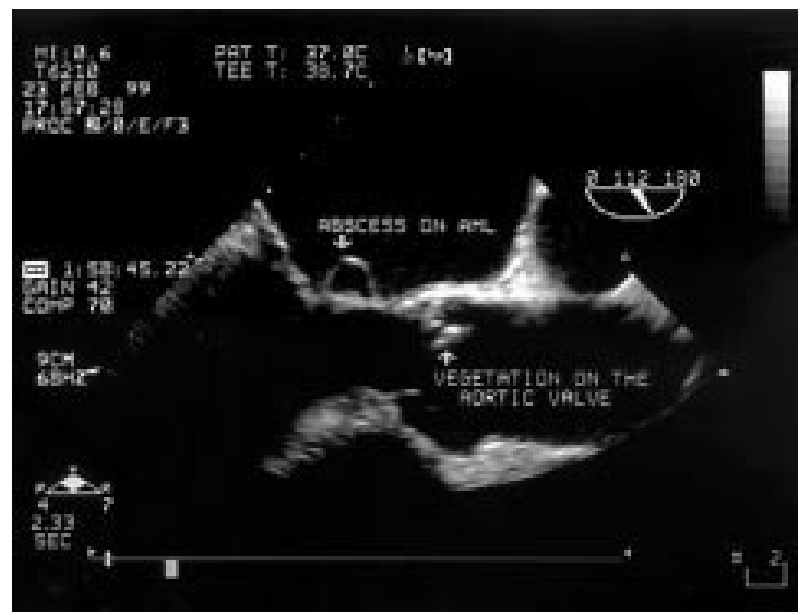

aortic valve and there was severe aortic regurgitation (below left). A transoesophageal echocardiogram demonstrated an abscess cavity on the atrial side of the anterior mitral leaflet where the mobile vegetation on the non-coronary cusp of the aortic valve was in contact with the mitral valve (below right).

At surgery the presence of a kissing abscess on the anterior mitral leaflet arising from a vegetation on the non-coronary cusp of the aortic valve was confirmed. St Jude's valve prostheses were implanted in the aortic and mitral positions. The patient made an uneventful recovery following surgery.

JACOB EASAW MAGDIE EL-OMAR MARK W RAMSEY

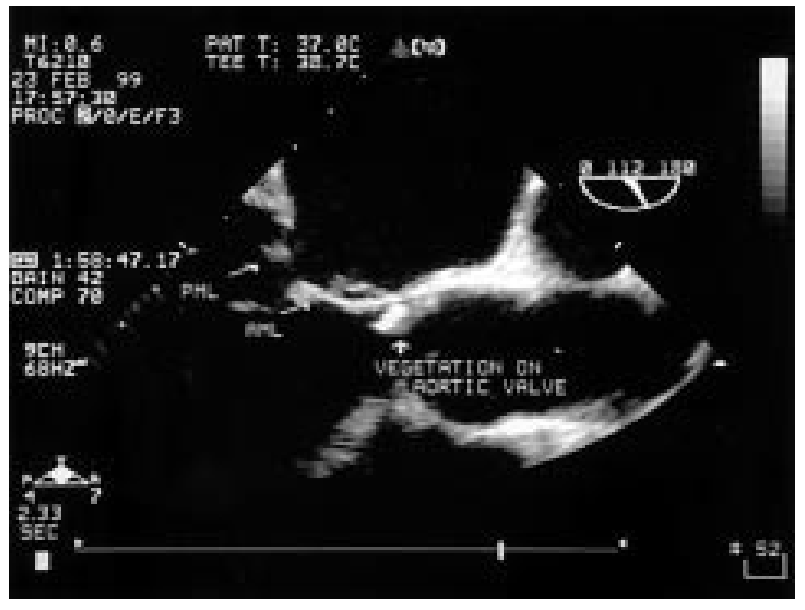

